\title{
Post-tuning of Sample Position in Common-path Swept-source Optical Coherence Tomography
}

\author{
Jae Seok Park, Myung Yung Jeong, and Chang-Seok Kim* \\ Department of Cogno-Mechatronics, WCU Program, Pusan National University, Busan 609-735, Korea
}

(Received August 22, 2011 : revised November 17, 2011 : accepted November 17, 2011)

\begin{abstract}
Common-path interferometers are widely used for endoscopic optical coherence tomography (OCT) because an arbitrary arm length can be chosen for the endoscopic imaging probe. However, the scheme suffers from the limited range of the sample position distance from the end of the imaging probe because the position between the reference reflector and the sample is limited by the optical path-length difference (OPD) to induce an interference signal. In this study, we developed a novel method for compensating the arbitrary sample position in common-path swept-source OCT by adding an extra Mach-Zehnder interferometer in the post-path of the interfered optical signal. Theoretical analysis and an experimental demonstration of imaging depth tuning for the flexible sample position of an endoscopic OCT image are discussed. After post-tuning of sample position distance, the positioning limitation between the reference reflector and the sample can be solved for various sample positions over a range of $26 \mathrm{~mm}$ for the cross-sectional images of a fish eye sample.
\end{abstract}

Keywords: Optical coherence tomography, Common-path interferometer, Biological imaging OCIS codes : (170.4500) Optical coherence tomography; (060.2380) Fiber optics sources and detectors; (060.0060) Fiber optics and optical communications

\section{INTRODUCTION}

Optical coherence tomography (OCT) is an emerging technology for non-invasive cross-sectional imaging of biomedical tissue samples [1]. It has been investigated for use in numerous applications, and thus, many types of system configurations have been developed. In most OCT systems, two beams are propagated to the reference arm and the sample arm and then re-coupled to produce an interfered optical signal [2, 3]. The general configuration of these two separate arms has limited the application of OCT systems in endoscopic imaging because an arbitrary probe length is required to deliver the optical signal from the internal organ tissue to the optical imaging system, which exists outside the body.

Recently, a common-path OCT system based on fiber-optic autocorrelation has been developed in order to overcome the length, polarization, and dispersion mismatch between the reference and sample arms [4-7]. Since there is only one path in common-path OCT, the reference and sample signals are reflected separately from the partial reflecting probe and the sample, respectively, and they propagate back through a commonly shared optical path [8]. However, a critical problem with conventional common-path OCT is that the sample position distance between the reference and sample positions is limited. The interfered optical signal between the reference and sample signals can be detected only when the delay time is within the coherence time of the light source. If the delay time between the reference and sample signals is $\tau_{\mathrm{S}}$ and the coherence time of the light source is $\tau_{\mathrm{C}}, \tau_{\mathrm{S}}$ should be shorter than $\tau_{\mathrm{C}}$ to produce an interfered optical signal. This means that the sample should be located inconveniently close to the reference position. As a result, unlike in the case of conventional Michelsonand Mach-Zehnder-type OCT, an objective lens with a long working distance cannot be utilized, and in-depth information about the sample cannot be obtained in this common-path OCT configuration. In the general OCT system, an objective lens with a low numerical aperture and a long working distance is commonly used to increase the penetration depth.

In this research, we developed a novel method for posttuning the optical path-length difference (OPD) between

\footnotetext{
*Corresponding author: ckim@pusan.ac.kr

Color versions of one or more of the figures in this paper are available online.
} 
the reference and sample signals for an arbitrary location of the reference and sample positions [4]. We present a novel common-path OCT configuration by including an additional Mach-Zehnder interferometer in the post-path of the interfered optical signal. Both 1-D profiles and 2-D images obtained with this novel common-path OCT and the conventional common-path OCT are compared. The OCT image is implemented with a Fourier domain OCT (FD-OCT) system based on a wavelength-swept laser source and a balanced detector [9-13].

\section{TUNING OF THE OPTICAL PATH LENGTH}

Fig. 1 shows a schematic of the novel common-path OCT system, in which the OPD is tuned by adding an extra interferometer in the post stage. A light beam from the wavelength-swept laser source propagates from the first port to the second port of the circulator and is transmitted through a probe fiber. The focused beam is reflected from the single-faced partial reflector and the sample, respectively. Then, it propagates to the third port of the circulator. Only one face of the partial reflector induces a small portion of the reflected light to act as the reference signal for the interferometer. The reference and sample signals share the common path of the fiber probe. There is a time delay, $\tau_{S}$, between the two signals from the single-face partial reflector and the sample. Assuming that the reference signal and sample signal are $E_{R}$ and $E_{S}$, respectively, the electric field $E_{3-p o r t}(t)$ at the third port of the circulator is given by

$$
E_{3-p o r t}(t)=\sqrt{R_{R}} E_{R}(t)+\sqrt{R_{S}} E_{S}\left(t+\tau_{S}\right)
$$

where $R_{R}$ and $R_{S}$ are the reflectivities of the partial reflector and the sample, respectively.

In general, the axial imaging depth is determined by the OPD between the reference and the sample. Because of the finite spectral resolution of the spectrometer in spectraldomain (SD) OCT or the instantaneous linewidth of the wavelength-swept laser in swept-source (SS) OCT, an increase

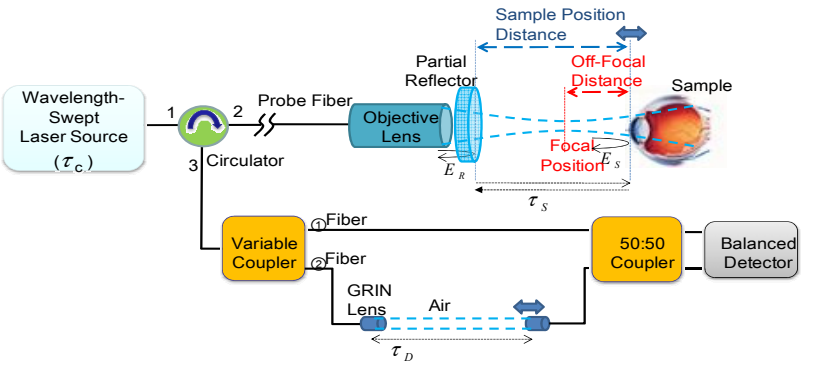

FIG. 1. Schematic of the novel common-path OCT system for tuning the optical path length. in the OPD leads to decay of the signal-to-noise ratio (SNR) and limits the range of the axial depth. Thus, in conventional common-path OCT, the more closely positioned partial reflector can be interfered to the sample with higher visibility signal. On the other hand, the OCT image also depends on the reflected optical intensity from the sample, which becomes maximal when the sample is located at the focal position of the objective lens. However, this results in a decreased SNR from the sample because the distance at the focal position of the objective lens corresponds to the increased OPD in the common-path OCT system.

To solve the above dilemma of the sample position, whether it be closer to the partial reflector or closer to the focal position, we suggest the addition of a Mach-Zehnder interferometer including a variable coupler, a tunable optical delay line (ODL), and a 50:50 coupler in the post-path of the interfered optical signal, as shown in Fig. 1. The signal from the third port of the circulator enters a variable coupler and propagates separately in two fiber paths (1), (2)). Then, those are combined at a 50:50 coupler. Therefore, the two terms on the right-hand side of equation (1) become four terms. When the splitting ratio of the variable coupler is $50 \%$, the electric field along paths (1) and (2) can be mathematically expressed as

$$
\begin{aligned}
& E_{1-\text { path }}=\frac{1}{2}\left(\sqrt{R_{R}} E_{R 1}(t)+\sqrt{R_{S}} E_{S 1}\left(t+\tau_{S}\right)\right) \\
& E_{2-\text { path }}=\frac{1}{2}\left(\sqrt{R_{R}} E_{R 1}\left(t+\tau_{D}\right)+\sqrt{R_{S}} E_{S 1}\left(t+\tau_{S}+\tau_{D}\right)\right)
\end{aligned}
$$

where $\tau_{D}$ denotes the additional delay time introduced by a tunable ODL.

Therefore, we can show that the total electric field detected at one of the balanced photodiodes becomes

$$
\begin{aligned}
\left|E_{\text {Total }}\right|^{2}= & \left|\frac{\sqrt{R_{R}} E_{R 1}(t)+\sqrt{R_{R}} E_{R 2}\left(t+\tau_{D}\right)+\sqrt{R_{S}} E_{S 1}\left(t+\tau_{S}\right)+\sqrt{R_{S}} E_{S 2}\left(t+\tau_{S}+\tau_{D}\right)}{2}\right|^{2} \\
= & \frac{1}{4}\left[\left|\sqrt{R_{R}} E_{R 1}\right|^{2}+\left|\sqrt{R_{R}} E_{R 2}\right|^{2}+\left|\sqrt{R_{S}} E_{S 1}\left(t+\tau_{S}\right)\right|^{2}+\left|\sqrt{R_{S}} E_{S 2}\left(t+\tau_{S}+\tau_{D}\right)\right|^{2}\right. \\
& +2\left\{\sqrt{R_{R}} E_{R 1}(t) \sqrt{R_{R}} E_{R 2}\left(t+\tau_{D}\right)+\sqrt{R_{R}} E_{R 1}(t) \sqrt{R_{S}} E_{S 1}\left(t+\tau_{S}\right)\right. \\
& +\sqrt{R_{R}} E_{R 1}(t) \sqrt{R_{S}} E_{S 2}\left(t+\tau_{S}+\tau_{D}\right)+\sqrt{R_{R}} E_{R 2}\left(t+\tau_{D}\right) \sqrt{R_{S}} E_{S 1}\left(t+\tau_{S}\right) \\
& \left.\left.+\sqrt{R_{R}} E_{R 2}\left(t+\tau_{D}\right) \sqrt{R_{S}} E_{S 2}\left(t+\tau_{S}+\tau_{D}\right)+\sqrt{R_{S}} E_{S 1}\left(t+\tau_{S}\right) \sqrt{R_{S}} E_{S 2}\left(t+\tau_{S}+\tau_{D}\right)\right\}\right]
\end{aligned}
$$

Among the combined terms with the reference and sample signals in the curly braces, it is clear that the term $\sqrt{R_{R}} E_{R 2}\left(t+\tau_{D}\right) \sqrt{R_{S}} E_{S 1}\left(t+\tau_{S}\right)$ can be the final interference signal in the case of $\left|\tau_{S}-\tau_{D}\right|<\tau_{D}$. This means that the post-tuning of the OPD in the additional Mach-Zehnder interferometer can induce the minimum OPD condition even when the sample is located at the focal position of the objective lens.

To determine the effect of the additional Mach-Zehnder interferometer, we can analyze the SNR of our proposed system. From the previous reports, SNR is defined as the 
signal power divided by the noise process variance $[5$, 14-16].

$$
S N R=\frac{\left\langle I_{a c}^{2}\right\rangle}{\sigma^{2}}
$$

where $\left\langle I_{a c}^{2}\right\rangle$ means the mean square signal photocurrent and $\sigma^{2}$ is the total noise variance.

Using each mean photocurrent and noise variance in a conventional system and in our system, our proposed system shows theoretically $6 \mathrm{~dB}$ lower sensitivity than the conventional system [5, 14-16]. However, our system is still promising because it provides flexible use of common-path probes by post-tuning of sample position distance. It means that this method is more suitable to obtain a clear cross sectional sample image at the long distance of sample position.

\section{EXPERIMENTAL CONDITION}

In this swept source OCT system, the output spectra from the wavelength-swept laser used is shown in Fig. 2(a). The laser has a fiber ring cavity configuration, with a semiconductor amplifier (SOA, InPhenix, Inc.), a piezo-actuated fiber FabryPerot tunable filter (FFP-TF, Lambda Quest, Inc.), an isolator, a polarization controller, and a fiber output coupler. The laser
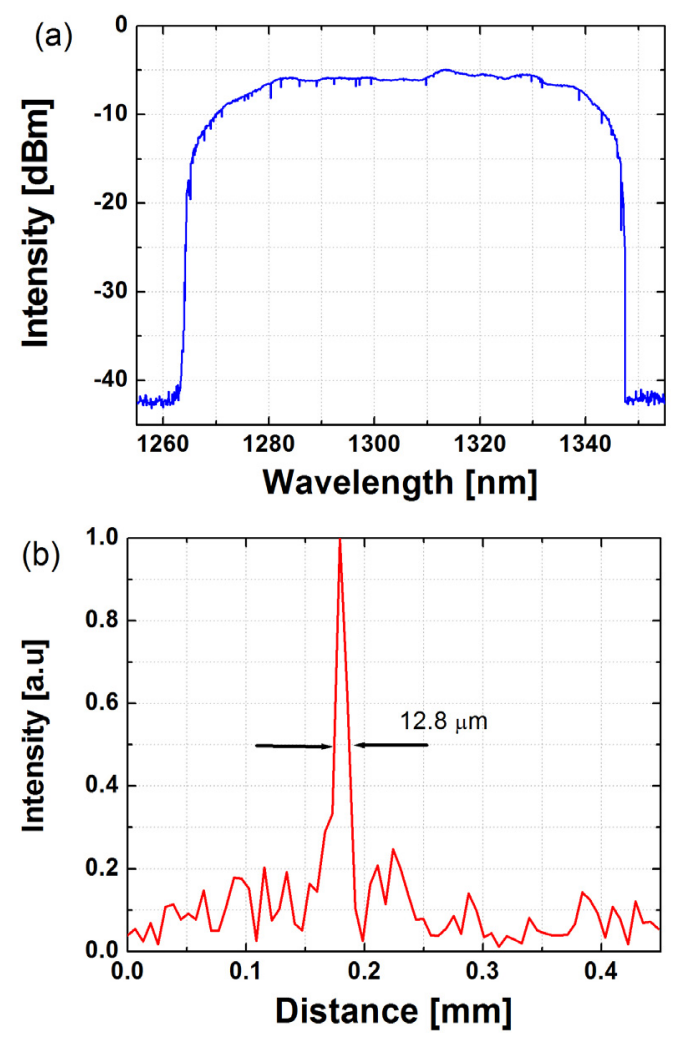

FIG. 2. (a) Output spectra from the FDML wavelength-swept laser; (b) axial resolution of the OCT system. output has a center wavelength of $1305 \mathrm{~nm}$, a bandwidth of $80 \mathrm{~nm}$, and an output power of $10 \mathrm{~mW}$. As we used a Fourier-domain mode locked (FDML) swept laser with a $2.8 \mathrm{~km}$ delayed fiber in the ring cavity, a high repetition rate of $71.09 \mathrm{kHz}$ is operated with stability. Fig. 2(b) shows the measurement result for an axial resolution of the OCT system processed by the Fourier transform of the acquired interferogram. The measured axial resolution is $12.8 \mu \mathrm{m}$ at the OPD of $1.8 \mathrm{~mm}$, which differs slightly from the theoretical value of $10 \mu \mathrm{m}$. It is thought that this difference can be attributed to the effect of dispersion introduced by the additional interferometer.

The objective lens, (model LSM03, magnification factor $=\mathrm{x} 5$, Thorlabs), has a focal length of $35.9 \mathrm{~mm}$ and a lens working distance of $25.1 \mathrm{~mm}$. Its transverse resolution is estimated to be $25 \mu \mathrm{m}$ from the mean spot size at the focal position. The partial reflector consists of two-sided glass. The antireflection-coated side has a reflectivity below 0.002 , and the other side has a reflectivity of 0.05 and a transmittance of 0.95 in the $1300 \pm 50 \mathrm{~nm}$ region. By posttuning the OPD, we could obtain OCT images, regardless of the distance between the partial reflector and the sample, within the long working distance of the objective lens. For the increased range of the sample position distance, the objective lens with lower magnification factor can be helpful to increase the working distance though the transverse resolution will be decreased.

An interferogram signal is detected by the balanced photodiode (model 1817, New Focus, USA), which has a bandwidth of $80 \mathrm{MHz}$, a trans-impedance gain of 50,000 V/A, and a responsivity of $0.8 \mathrm{~A} / \mathrm{W}$ at $1300 \mathrm{~nm}$. By dual balancing, the DC level is suppressed and no additional background subtraction is conducted. Acquired interferogram data are transferred to a personal computer via a data-acquisition board (PCI-5124, National Instruments Corp., USA), which has a maximum sampling rate of $200 \mathrm{Msamples} / \mathrm{s}$ for the analog signal. Fast Fourier transformation is performed on the transferred data. To acquire a single cross-sectional image, a linear translation stage is driven with a saw-tooth waveform to provide transverse scanning over a $3 \mathrm{~mm}$ distance along the sample surface. General LabVIEW software is used to process the image.

\section{EXPERIMENTAL COMPARISON}

To discriminate between the absolute distance (sample position distance) and the relative distance (OPD) under the same conditions, we fixed the location of the partial reflector at $6 \mathrm{~mm}$ from the focal position, as shown in Fig. 1, by the repeated optimization process of the reference position for the maximized SNR [4]. Within the lens working distance of the objective lens, which is $25.1 \mathrm{~mm}$, the position of the mirror sample is changed along the axial beam distance to measure a series of point-spread functions (PSFs). We call this distance between the focal position and the sample 
surface the "off-focal distance."

First, for the conventional common-path OCT, the dynamic range roll-off is measured in Fig. 3 as the off-focal distance is changed incrementally. The off-focal distance range $0-9 \mathrm{~mm}$ corresponds to both "sample position distance" and "OPD" range of $6-15 \mathrm{~mm}$ because the interfered optical signal by the OPD is exactly the same with the sample position distance between the partial reflector and the sample in this conventional common-path OCT. The dynamic range is determined by the ratio of the PSF peak value and the noise level. The noise level is determined to be $-5 \mathrm{~dB}$ by averaging the region between 0 and $9 \mathrm{~mm}$. The system dynamic range drops rapidly as the off-focal distance increases. As can be seen in Fig. 3(a), the dynamic range drops rapidly from 35.9 to $7.6 \mathrm{~dB}$ by increasing the off-focal distance from 0 to $10 \mathrm{~mm}$. The $6 \mathrm{~dB}$ drop out is measured at off-focal distance of around $6 \mathrm{~mm}$. As mentioned in the previous section, this rapid roll-off of $27.9 \mathrm{~dB}$ is due to two reasons. First, the finite sample number due to the thick instantaneous linewidth in the swept source leads to strong decay of the SNR for a deeper axial range of the incremental OPD. Second, the signal beam has weak reflectivity as the sample moves away from the focal position $[9,10]$. Therefore, when imaging with the conventional common-path OCT, the sample should be located as close as possible to the partial reflector at
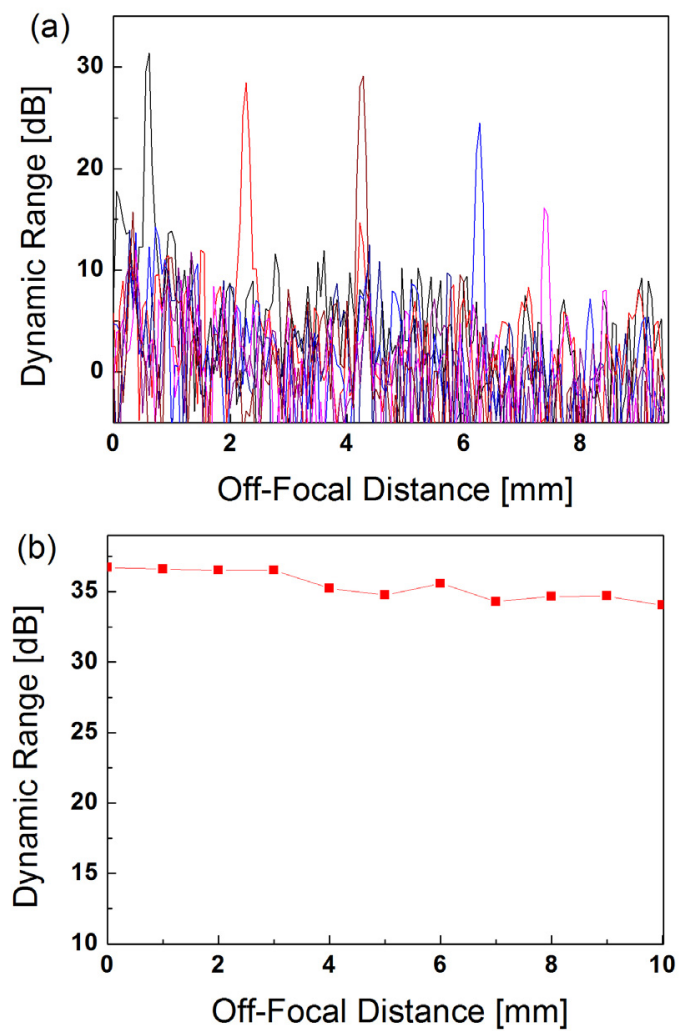

FIG. 3. Dynamic range determined by locating the mirror sample at various off-focal distances (a) without post-tuning the OPD and (b) by setting the PSF peak at the OPD near 0 mm and tuning $\tau_{\mathrm{D}}$ to be the same as $\tau_{\mathrm{S}}$. around the focal position of the objective lens. These two requirements may restrict the available position of the sample significantly in the limited range.

In Fig. 3(b), for the purpose of comparison, the dynamic range is measured again using the novel common-path OCT setup by locating the mirror sample at the same off-focal distances from 0 to $9 \mathrm{~mm}$. At every off-focal distance, the extra delay time, $\tau_{\mathrm{D}}$, of the fiber path (2) can be manually tuned to be the same as the original delay time, $\tau_{\mathrm{S}}$, and thus, the PSF peak can be set at the OPD near $0 \mathrm{~mm}$ repeatedly. The recovery of the dynamic range is clearly monitored from the control of the OPD so that the dynamic range drops slightly from 36.9 to $34.2 \mathrm{~dB}$ when the off-focal distance is increased from 0 to $10 \mathrm{~mm}$. It seems that this slight roll-off of $2.7 \mathrm{~dB}$ is due to one reason: the sample signal has weak reflectivity when the sample is away from the focal position. It also means that the effect of the focal position on the quality of the OCT image is relatively weaker than that of the OPD. Moreover, the effect of the OPD can be ignored because the sample position distance between the partial reflector and the sample is compensated by tuning the path length by $\tau_{\mathrm{D}}$. This shows that our novel common-path OCT system can be just as useful as the other two separate-path OCT systems on the distance flexibility of sample position. We expect the slight fluctuation of PSF peak values in Fig. 3 can be suppressed when we further improve the axial alignment of optical components and the feedback control of the manual OPD compensation.

Dynamic range can also be compared for various samples by using the conventional and novel common-path OCT systems. Fig. 4 shows the depth profiles of a sample consisting of two slide glasses acquired at different sample position distances between the partial reflector and the sample using a conventional common-path OCT system. When the top surface of the sample is positioned close to the partial reflector at $0.3 \mathrm{~mm}$ (Fig. 4(a)), we can observe the two glasses and the air gap between them clearly. As the sample position distance increases, the image quality is strongly degraded because of the dynamic range roll-off. When the distance between the partial reflector and the top surface of the sample becomes more than $3.3 \mathrm{~mm}$, the top surface of the upper glass can be monitored with difficulty, the other surfaces not at all. It means that there is no way to get a good-quality image in the conventional common-path OCT system without positioning the sample close to the partial reflector.

For the additional comparison with a cross sectional OCT imaging, the biological sample of a fish eye (Pangasius sutchi) is also imaged in our proposed swept-source common-path OCT system by changing the sample position distance, as shown in Fig. 5. For the much extended range of sample position distance, we used the other objective lens, (model LSM04, magnification factor $=x 3$, Thorlabs), with a focal length of $53.9 \mathrm{~mm}$ and a lens working distance of $42.3 \mathrm{~mm}$. When the sample surface was placed at the focal position and the partial reflector was located at $6 \mathrm{~mm}$ from the 


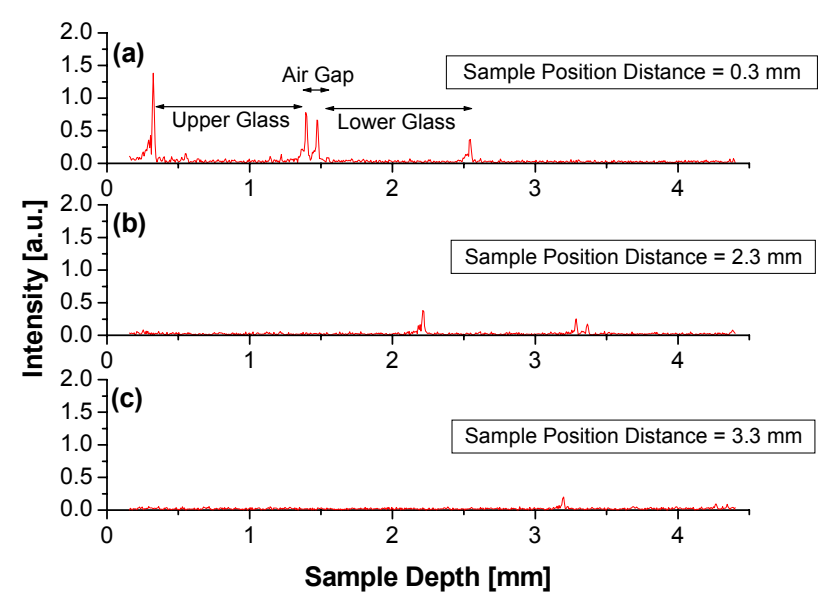

FIG. 4. Depth profiles of two slide glasses obtained with the conventional common-path OCT system for different sample position distances: (a) $0.3 \mathrm{~mm}$, (b) $2.3 \mathrm{~mm}$, and (c) $3.3 \mathrm{~mm}$.

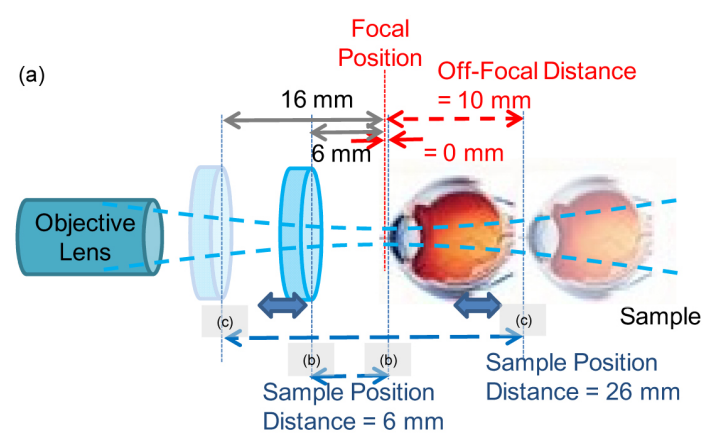

(b) Sample Position Distance

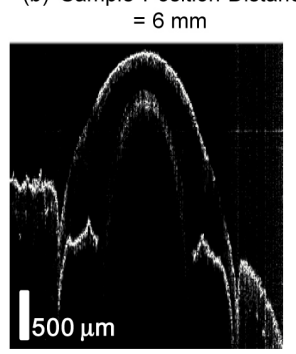

(c) Sample Position Distance

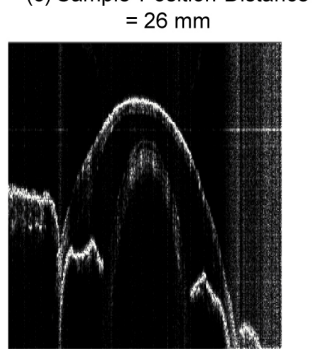

FIG. 5. (a) Setup for cross-sectional images of a fish eye obtained with the proposed common-path OCT system for different sample position distances and the OCT images for the sample position distance of (b) $6 \mathrm{~mm}$ and (c) $26 \mathrm{~mm}$, respectively.

focal position as shown in Fig. 5 (a), the inner structure of eye sample can be clearly observed as shown in Fig. 5 (b). For the estimate the maximum available range of sample position distance, we moved the partial reflector toward the objective lens and the sample in the opposite direction, simultaneously, up to $10 \mathrm{~mm}$ for both sides. As the sample moves away from the reference position, we also increase the extra delay time, $\tau_{\mathrm{D}}$, to move the highest PSF peak toward the $0 \mathrm{~mm}$ point of the OPD. As shown in Fig. 5 (c) at the sample position distance of $26 \mathrm{~mm}$, the quality of OCT image becomes degraded because the beam intensity and transverse resolution are relatively decreased due to the long distance from the focal position. The effect of intensity degradation is as low as $2.7 \mathrm{~dB}$ for the off-focal distance of $10 \mathrm{~mm}$, as shown in Fig. 3 (b). The mean spot size of this objective lens is $35 \mu \mathrm{m}$ at the focal position and the transverse resolution can be estimated to $\sim 100 \mu \mathrm{m}$ at the off-focal distance of $10 \mathrm{~mm}$. This means that there is a negligible degradation in the axial image quality up to the sample position distance of $26 \mathrm{~mm}$, as predicted in Fig. 3(b), and the distance problem between the partial reflector and the sample can be solved for the practical endoscopic applications. The image size is $3 \mathrm{~mm} \times 3 \mathrm{~mm}$, which consists of 256 axial scans (transverse pixels) with 256 points (axial pixels) per scan. In case of a conventional common-path OCT system, as expected from the results of strong fall-off in Fig. 4, it is monitored that image quality is seriously degraded at a few $\mathrm{mm}$ of the sample position distance. Moreover optical interference does not occur at more than $\sim 20 \mathrm{~mm}$ of sample position distance.

\section{CONCLUSION}

We have developed a novel method for post-tuning the sample position distance in a common-path OCT system by including an additional Mach-Zehnder interferometer in the post-path of an interfered optical signal. From the experimental results of post-imaging depth tuning, the dynamic roll-off of the system is reduced to be as low as $2.7 \mathrm{~dB}$ during $10 \mathrm{~mm}$ axial scanning. This can solve the positioning problem for the objective lens, partial reflector, and sample in the conventional common-path OCT system. The proposed method may allow for greater flexibility in the use of OCT systems in endoscopic applications.

\section{ACKNOWLEDGMENT}

This work was supported for two years by Pusan National University Research Grant.

\section{REFERENCES}

1. D. Huang, E. Swanson, C. Lin, J. Schuman, W. Stinson, W. Chang, M. Hee, T. Flotte, K. Gregory, C. Puliafito, and J. Fujimoto, "Optical coherence tomography," Science 254, 1178-1181 (1991).

2. R. C. Youngquist, S. Carr, and D. E. N. Davies, "Optical coherence-reflectometry: a new optical evaluation technique," Opt. Lett. 12, 158-160 (1987).

3. K. Takad, I. Yokohama, K. Chida, and J. Noda, "New measurement system for fault location in optical waveguide devices based on an interferometric technique," Appl. Opt. 26, 1603-1606 (1987). 
4. U. Sharma, N. M. Fried, and J. U. Kang, “All-fiber commonpath optical coherence tomography: sensitivity optimization and system analysis," IEEE J. Select. Topics Quantum Electron. 11, 799-805 (2005).

5. J. U. Kang and A. Rodrigues, "Fourier domain common-path fiber OCT with tunable reference: analysis and optimization," in Proc. CLEO-QELS 2007 (Baltimore, USA, May 2007), JTuA55.

6. A. B. Vakhtin, D. J. Kane, W. R. Wood, and K. A. Peterson, "Common-path interferometer for frequency-domain optical coherence tomography," Appl. Opt. 42, 6953-6958 (2003).

7. A. R. Tumlinson, J. K. Barton, B. Povazay, H. Sattman, A. Unterhuber, R. A. Leitgeb, and W. Drexler, "Endoscope-tip interferometer for ultrahigh resolution frequency domain optical coherence tomography in mouse colon," Opt. Express 14, 1878-1887 (2006)

8. J. S. Park, M. Y. Jeong, C. H. Jung, C. H. Ouh, H. J. Kang, Y. G. Han, S. B. Lee, and C. S. Kim, "Flexible curled optical cord for bending-insensitive optical imaging delivery," IEEE J. Select. Topics Quantum Electron. 16, 1031-1038 (2010).

9. S. H. Yun, G. J. Tearney, J. F. de Boer, and B. E. Bouma, "Removing the depth-degeneracy in optical frequency domain imaging with frequency shifting," Opt. Express 12, 4822-4828 (2004).
10. J. F. de Boer, B. Cense, B. H. Park, M. C. Pierce, G. J. Tearney, and B. E. Bouma, "Improved signal to noise ratio in spectral-domain compared with time-domain optical coherence tomography," Opt. Lett. 28, 2067-2069 (2003).

11. Y. Nakamura, S. Makita, M. Yamanari, M. Itoh, T. Yatagai, and Y. Yasuno, "High-speed three-dimensional human retinal imaging by line-field spectral domain optical coherence tomography," Opt. Express 15, 7103-7116 (2007).

12. J. H. Lee, E. J. Jung, and C. S. Kim, "Optical coherence tomography based on a continuous-wave supercontinuum seeded by erbium-doped fiber's amplified spontaneous emission," J. Opt. Soc. Korea 14, 49-54 (2010).

13. R. Huber, M. Wojtkowski, K. Taira, and J. G. Fujimoto, "Amplified, frequency swept lasers for frequency domain reflectometry and OCT imaging: design and scaling principles," Opt. Express 13, 3513-3528 (2005).

14. R. Leitgeb, C. K. Hitzenberger, and A. F. Fercher, "Performance of fourier domain vs. time domain optical coherence tomography," Opt. Express 11, 889-894 (2003).

15. A. M. Rollins and J. A. Izatt, "Optimal interferometer designs for optical coherence tomography," Opt. Lett. 24, 1484-1486 (1999).

16. M. A. Choma, M. V. Sarunic, C. Yang, and J. A. Izatt, "Sensitivity advantage of swept source and Fourier domain optical coherence tomography," Opt. Express 11, 2183-2189 (2003). 